\title{
Numerical Modelling of Light Propagation for Development of Capillary Electrophoretic and Photochemical Detection Systems
}

\author{
Tomasz Piasecki, Aymen Ben Azouz, Brett Paull, \\ Mirek Macka and Dermot Brabazon
}

Additional information is available at the end of the chapter

http://dx.doi.org/10.5772/45764

\section{Introduction}

Capillary electrophoresis (CE) has been used for over 30 years as an efficient separation technique [1]. These separations are typically preformed using capillaries with internal diameter ranging from $2 \mu \mathrm{m}$ to $200 \mu \mathrm{m}$ and as the diameter increases above $100 \mu \mathrm{m}$, a reduction in separation performance is observed [2]. One of the main factors limiting the overall performance of CE separations is Joule heating associated with the passing of the electric current through resistive medium. The high surface-to-volume ratio in a smaller capillary diameter allows for efficient heat dissipation which is beneficial for compound separation, however, simultaneously this has a negative impact on the detection performance due to the corresponding reduction in analyte volume available for detection [3].

Historically when capillaries were first used for CE, they were coated with polyimide and presented excellent robustness but posed problems with optical detection as polyimide is highly absorbing below 550nm preventing optical detection in that spectral range. Such capillaries were striped of the coating to enable the optical detection in UV and low wavelength visible range. However, capillaries striped of their coating are brittle and can easily be damaged making usage often impractical. The introduction of the polytetrafluoroethylene (PTFE) coated capillaries allowed for optical detection within the UV range as well as across the entire visible spectrum. Although the PTFE coated capillaries are not as durable as the polyimide coated capillaries, they are significantly more robust than coatless ones and allow for easier deployment during typical daily laboratory routine. 
Absorbance photometry is a detection technique used to determine the concentration of target species in a liquid sample based on interaction between the probe light and species [4]. Typically it is a measurement of the light intensity with and without a sample placed in the light path. A scheme of light intensity measurement with the sample located for detection is presented in Figure 1. The sample transmittance, $T$, is defined as the ratio of the initial light intensity, $I_{0}$, to the recorded light intensity, $I_{1}$ (Eq. 1). Io should be measured with the sample holding cuvette empty. This allows for reflections and potential absorption by the cuvette material to be taken into account during sample measurement. Sample absorbance, $A$, is measured as the negative log of the transmittance (Eq. 2). The cuvette length $l$ is known, as well as species molar absorptivity coefficient $\alpha$, which is a specific characteristic of every species. Light attenuation along the light path is governed by BeerLambert's law where $c$ is molar concentration (Eq. 3) [5]. The method of calculating the actual optical path length is presented elsewhere [6].

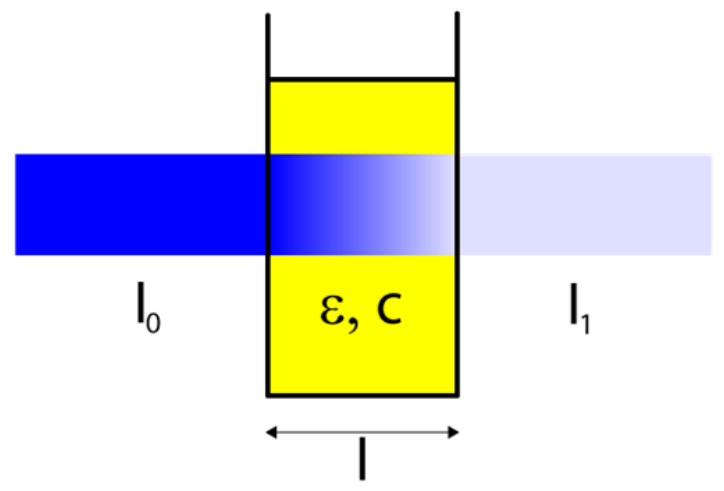

Figure 1. Schematic of light intensity with sample located for photometric detection

$$
\begin{gathered}
T=\frac{I_{1}}{I_{0}} \\
A=-\log T=-\log \frac{I_{1}}{I_{0}} \\
I(l)=I_{0} \cdot e^{-\alpha c l}=I_{0} \cdot e^{-A}
\end{gathered}
$$

Despite excellent performance in analyte separation, CE techniques still constitute a minority of the commercial applications due to detection limitations imposed by popular absorbance photometric detectors. Various developments in CE have occurred such as the development of UV-transparent capillaries which facilitated CE by making it possible to employ the commercially available detectors. In the past different approaches have also been employed to alter the geometry of the detection system in order to improve the detection performance for $\mathrm{CE}$. These include the application of rectangular capillaries to reduce refraction effects on the cylindrical boundaries [2], multi-reflection cells for increased signal intensity [7], and development of Z-shaped flow cells with increased optical pathlength for analyte detection [8]. Some detectors were built employing these methods, 
but to date the majority of the commercially available absorbance based detectors work on the basis of a single passage of light through the sample contained in a capillary.

To gain a better understanding of light propagation through capillaries and how to improve detection levels, the modelling of the light propagation through capillaries has been previously undertaken. Much of this work was limited to two-dimensional projections and two-layer models (no coating present) taking into account capillary material and inner cavity [9]. Previous numerical simulations of light propagation through capillaries have been reported as a tool for flow-cell design and have been limited to prediction of light path for these specific cases only [10]. The numerical problem of ray-tracing through a capillary is an excellent example where advantage can be taken of the ability to perform numerous calculations quickly on a computer to allow for ray paths, ray path overlaps, and resultant light intensities to be calculated.

This book chapter presents a theoretical study on the light propagation through coated capillaries, focusing on PTFE-coated capillaries. These models can be used to increase the performance of absorbance photometric detection and for associated photochemical applications.

\section{General description of the model}

The Light propagation and light intensity distribution models were developed using LabVIEW $^{\mathrm{TM}} 2011$ graphical programming environment. Graphic-related work, such as generation of multi-colour maps and reading pixel colours were conducted with Adobe Photoshop CS 3 ver. 10.0.1. Dimension and angle measurements taken from photos were performed using Image J $1.43 \mathrm{u}$ software.

Whenever light is incident on the boundary of two transparent dielectrics part of it is reflected and part is transmitted, see Figure 2. The angle of incidence is related with the angle of transmittance by Snell's Law (Eq. 4). In the case of cylindrical symmetry, as in capillaries, it is possible that the exiting ray will not be parallel with the incident, see Figure 3.

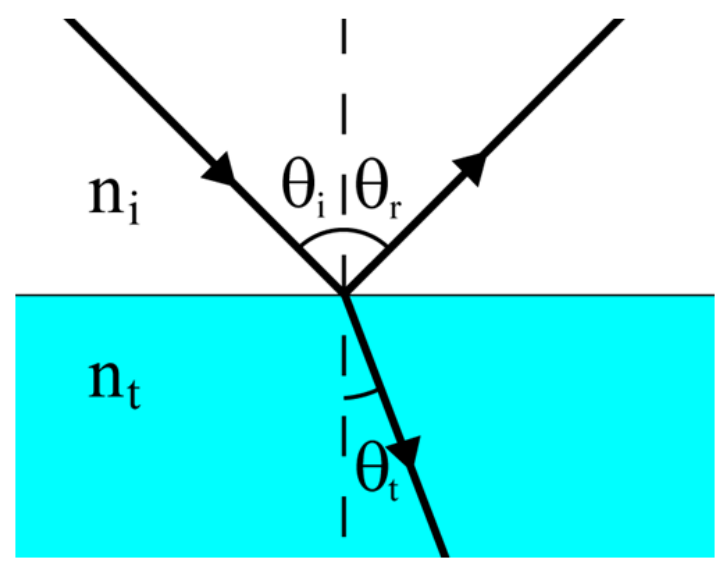

Figure 2. Light incident on boundary of two different dielectrics. 

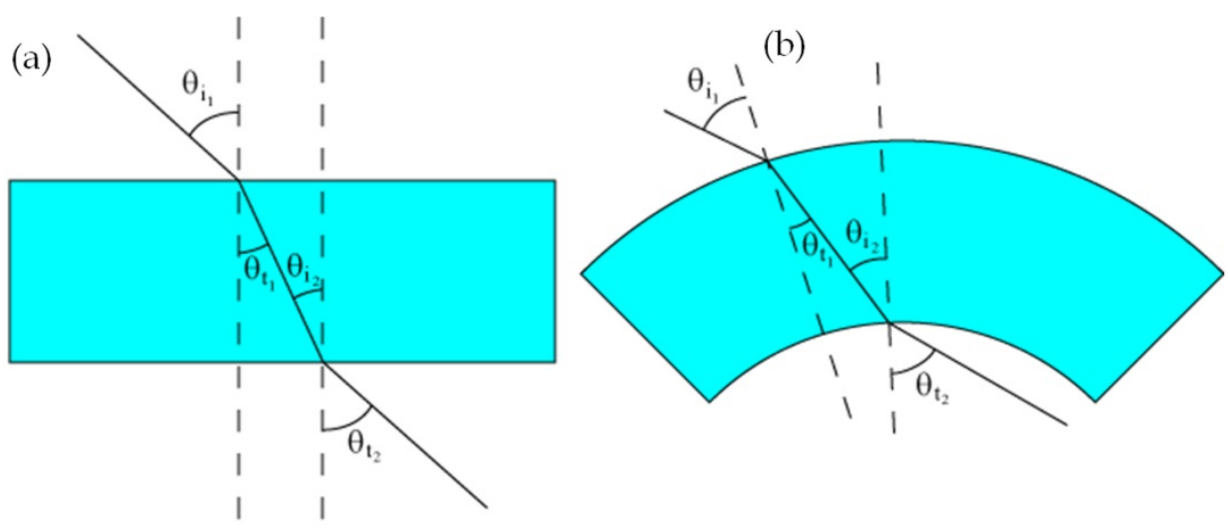

Figure 3. Schematic of light transmission through, (a) a flat transparent material and (b) a cylindrical capillary, showing curvature affects on angle of incidence and transmittance.

$$
n_{i} \cdot \sin \theta_{i}=n_{t} \cdot \sin \theta_{t}
$$

where $n_{i}$ is the refractive index of the incident ray transmission medium, $\theta_{i}$ is the angle between incident wavevector and the normal to surface, $\mathrm{n}_{t}$ is the refractive index of the material through with the transmitted ray passes and $\theta_{t}$ is the angle between transmitted wavevector and normal to the surface. Light attenuation inside the absorbing medium is governed by Beer-Lambert law (Eq. 5).

$$
I=I_{0} \cdot e^{-\alpha c l}
$$

where I is the initial light intensity, Io is the light intensity behind the sample, $\alpha$ is the molar absorptivity coefficient, $\mathrm{c}$ is concentration of the compound and $l$ is length is the absorption path length.

The numerical modelling software was developed to calculate the light ray path through multi-layered cylinders, and the light intensity distribution map through the cylinder cross-sectional area. Light propagation within multimode optical fibres occurs by the phenomena of total internal reflection, where the values of refractive indices and fibre diameter remain within the limits of geometrical optics. The size of the capillary used in this work was comparable with the size of multimode optical fibres. It was assumed that capillary body, coating and bore were perfectly cylindrical and concentric. Only the right half of the capillary cross-section is displayed in the developed model, as the diameter acts as the modelled axis of symmetry and no light ray could propagate through from left to right side. In general such occurrence is possible, but only for higher values of refractive indices approximately twice those of glass and PTFE which were used in this work.

The programmed model calculated the light ray path equations in the Cartesian coordinate system. Separate linear functions to describe each of the light ray path segments were used (for example between air/tube, tube/tube or tube/liquid). 

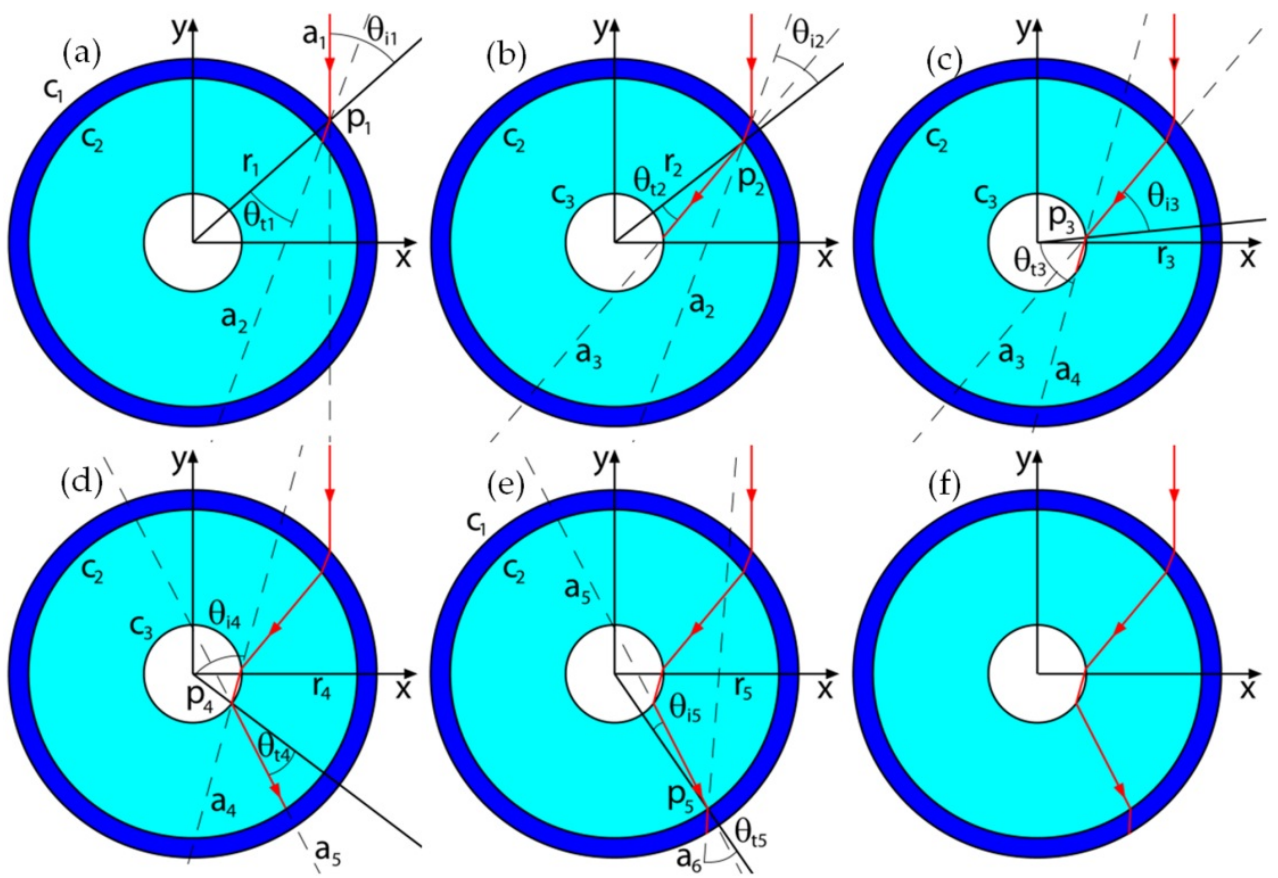

Figure 4. Schematic of reference lines and points, and light ray path calculated by each sub-routine for light passing through a three layer system in which the light ray path is passing through a coated hollow capillary.

Each light path segment was calculated in a separate subroutine calculating the light path in each zone. Incident light was in the form of rays parallel to y-axis, see Figure 4 . The first subroutine calculated the coordinates of the light incident from infinity on the air/coating boundary under desired angle. This point of incidence of the ray a1 on outermost circle $\mathrm{c} 1$ was assigned as $\mathrm{p}_{1}$. An extended radius $\mathrm{r}_{1}$ going from $(0,0)$ through $\mathrm{p}_{1}$ was drawn for calculation of angle of incidence $\theta_{\mathrm{i} 1}$ and in turn angle of transmission $\theta_{t 1}$ was calculated from $\mathrm{Eq} .4$, for the use inputted values of refractive indices $\mathrm{n}_{i}$ and $\mathrm{n}_{t}$. A line a2 through $\mathrm{p}_{1}$ was drawn representing the refracted ray in the coating with $\theta_{t 1}$ as the angle between $\mathrm{a}_{2}$ and $\mathrm{r} 1$, ending the first program subroutine, see Figure 4A. The next subroutine began with calculation of point $\mathrm{p}_{2}$ (where line a2 crossed boundary $\mathrm{c}_{2}$ ) and the drawing of extended radius $\mathrm{r}_{2}$ from $(0,0)$ through $\mathrm{p}_{2}$. Angle of incidence $\theta_{\mathrm{i} 2}$ was calculated as the angle bounded by $\mathrm{r}_{2}$ and a2, see Figure $4 \mathrm{~B}$. Angle of transmittance $\theta_{t 2}$ was calculated from Eq. 4 and the line a3 was drawn where $\theta_{t 2}$ was and angle between $\mathrm{r}_{2}$ and $\mathrm{a}_{3}$, ending the second program subroutine, see Figure $4 \mathrm{~B}$. This subroutine was iterated a further three times to calculate light ray paths segments along lines $\mathrm{a}_{4}$, $\mathrm{a}_{5}$ and $\mathrm{a}_{6}$ after refraction on each encountered boundary. These next subroutines of the light path formation are illustrated in Figure $4 \mathrm{C}$ to $\mathrm{E}$ and the complete generated light path is presented in Figure $4 \mathrm{~F}$ without reference lines. The entire light path was represented as a sum of individual rays calculated separately according to the symbolic algorithm: 


$$
r=\sum_{k=1}^{2 n}\left(a_{k} \cap c_{k} \rightarrow p_{k} \rightarrow r_{k} \rightarrow \theta_{i_{k}} \rightarrow \theta_{t_{k}} \rightarrow a_{k+1}\right)
$$

where $\mathrm{r}$ is a light ray path, $\mathrm{k}$ is a step number; $\mathrm{n}$ is a number of layers; $\mathrm{a}, \mathrm{c}, \mathrm{p}, \mathrm{r}, \theta_{\mathrm{i}}$ and $\theta_{t}$ are as described earlier. The experiments on model validation and their results are presented elsewhere [11].

\section{Application of the developed model for optimisation of the capillary- based optical detection system}

The main aim of this part is to present quantitative information about light behaviour in capillary base detection setups. Their highly complicated geometry and multi-layered construction results in unusual optical properties can significantly affect the performance of the detection system if not designed and manufactured carefully.

The light attenuation inside light absorbing medium is governed by Lambert-Beer's law (Eq. 2). However in this form it is assumed that the light that is collected by a detector is concurrent with the light that passed through the absorbing medium. In real situation there is additional component that can seriously affect photometric measurements: the stray light. It can be defined as the light that omits the sample but still is collected by the detector. In such case (Eq. 2) should be written in another form (Eq.4):

$$
A=-\log \frac{I_{A}-I_{S}}{I_{0}}
$$

where: A is absorbance, $I_{A}$ is the light intensity of the attenuated light beam, Is is intensity of the stray light, and $\mathrm{I}_{0}$ is the initial light intensity. In such case, with increase of the amount of light attenuating medium IA goes down to zero and Eq. 6 becomes Eq. 7, effectively imposing maximum possible absorbance:

$$
A=-\log \frac{I_{S}}{I_{0}}=\text { const }
$$

Figure 5 presents the maximum possible absorbance recorded depending on the percentage amount of the stray light in the detection system. For the illustration purpose, the relation between concentration and absorbance is set to one. The line labelled "Ideal" represents situation with no stray light at all, giving completely linear response depending on the test compound concentration. The other four curves labelled " $10 \%$ ", " $1 \%$ ", " $0.1 \%$ " and " $0.01 \%$ " represent the same response with the respective percentage of the stray light present in the system. This allows visualising the detection limits associated with the imperfectness of the detection system. Also it is noteworthy to compare the deviation from the linearity that is associated with the stray light.

Figure 6 presents the deviation from the ideal response depending on the amount of the stray light. As it can be read from the graph on Figure 6, the deviation is small, and therefore assumption of linearity is valid typically one absorbance unit before the maximum, e.g. for 
$0.1 \%$ of the stray light, the maximum measure absorbance is $3 \mathrm{AU}$, and the assumption of linearity is valid from 0 to 2 AU.

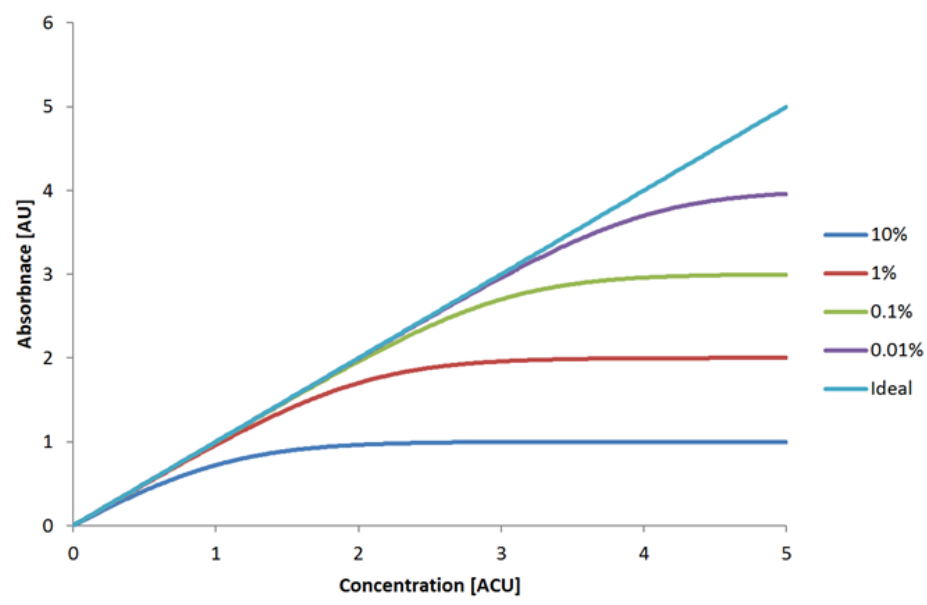

Figure 5. A comparison of different amounts of stray light present in the detection system and how it imposes detecion limits. Concentrations are scaled in arbitrary concentration units (ACU). Absorbance scaled in absorbance units (AU).

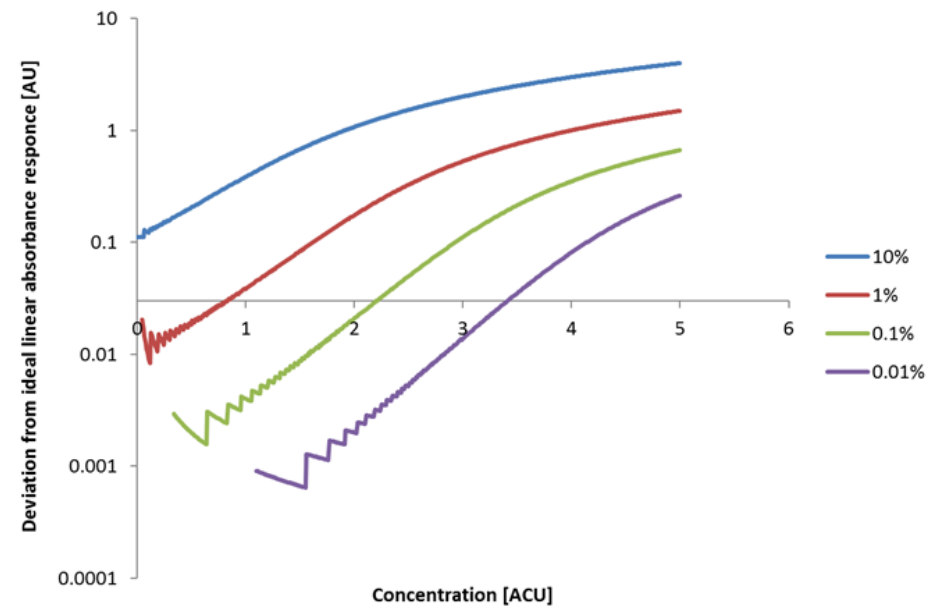

Figure 6. A comparison of the deviation from the linear response depending on the amount of stray light in the system. Concentration scaled in arbitrary concentration units (ACU). Absorbance scaled in absorbance units (AU).

For numerous reasons it is convenient to employ the same capillary for detection after any reactions or separations. The cylindrical geometry of the capillary combined with its multilayer structure creates a quite complex medium for the light to propagate through and eventually can significantly alter the light path. Figure 7 presents a simplified image of 
possible light refractions on different boundaries (air/coating, coating/fused silica body, body/content). In this figure only light perpendicular to the capillary symmetry axis is portrayed. In real situation light coming out from an uncollimated light source can be incident under any angle. This situation can be compared with a capillary illuminated by a laser.

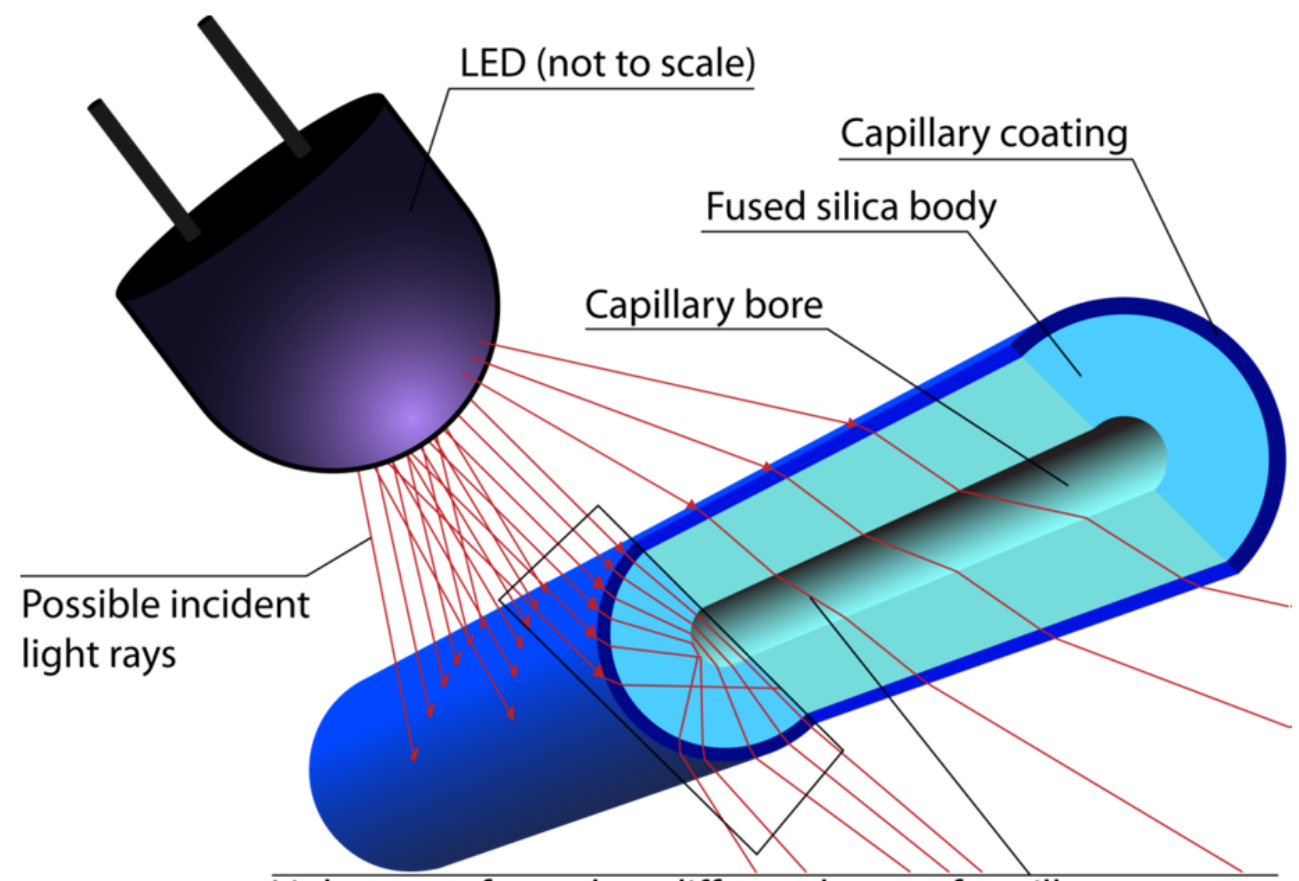

Light rays refracted on different layers of capillary structure

Figure 7. Schematic of a capillary cross-section illuminated by a beam of parellel rays.

In order to prevent undesired light to reach the detector the light source and the detector should be comparable with the capillary bore internal diameter. This can be achieved by installing an aperture or by using an optical fibre to illuminate the capillary and collect the light for detection. Such application of optical fibres has been reported already several years ago [12], [13]. Application of any of these two approaches has an impact on the overall performance of the detection system. Light going through an aperture undergoes diffraction and light coming out of an optical fibre is divergent with the spread angle confined by numerical aperture (NA) of the fibre itself. The NA of 0.22 gives cone of acceptance with maximum possible angle of $25.4^{\circ}$, being as well the maximum angle at which the light will exit the fibre. Figure 8 present a comparison of the light intensity that can interact with capillary bore content depending on the distance at which a $100 \mu \mathrm{m}$ collecting optical fibre of with NA of 0.22 for four different maximum incidence cones. Zero degrees is equivalent of an ideally parallel beam, while 25 degrees is an equivalent of the light coming out of an optical fibre. Light intensity loss is the effect of the capillary geometry only and the capillary coating, 
capillary body and the theoretical solution filling the capillary bore are assumed to be completely transparent. As it can be seen the light coming out of the optical fibre has the angle of divergence of 25 degrees. This results that only over $22 \%$ of the delivered light intensity can reach the capillary bore, interact with its content and be collected by similar $100 \mu \mathrm{m}$ diameter optical fibre. That number decreases with distance as light after propagating through the entire capillary becomes even more divergent, similarly to a ball lens.

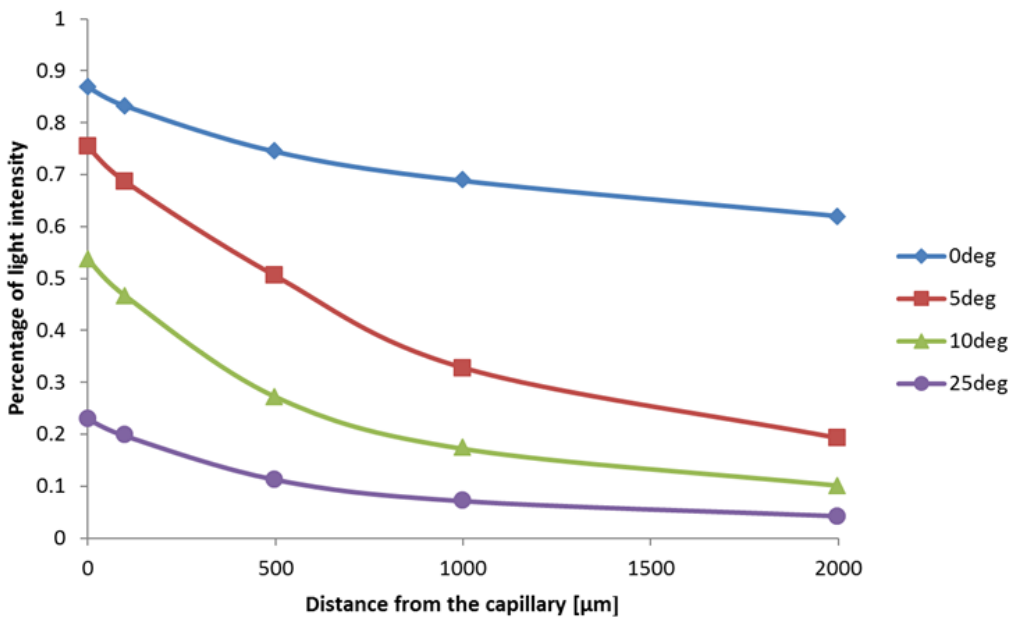

Figure 8. Relation between the percentage of light passing though the capillary bore, comming from a $100 \mu \mathrm{m}$ fibre, and the distance between the collecting $100 \mu \mathrm{m}$ optical fibre and the capillary. These results were calculated for incident beams with $0,5,10$ and 25 degrees divergent cone of illumination.

The light intensity loss associated with the divergent illuminating beam is not the only concern. At the same time due to the capillary geometry it is possible that the light exiting the illuminating fibre will enter the capillary, propagate through omitting the capillary bore completely and exit it in a manner that can still enter the collecting optical fibre. Such light would contribute toward the stray light and reduce overall efficiency of the detection system. Figure 9 illustrates the percentage amount of the stray light that can enter collecting optical fibre under an angle below the numerical aperture depending on a distance between the capillary and the end of the fibre.

For the collecting fibre perfectly touching the capillary the amount of stray light is zero. However already at distance of $0.5 \mathrm{~mm}$ this goes up to $40 \%$ if the capillary is illuminated with an optical fibre as well. The observed maximum for 10 degrees divergent incident beam (Figure 9 - green line) followed by drop of stray light to zero a $2 \mathrm{~mm}$ from capillary is a result of the capillary geometry - light omitting the capillary bore after passing the coating and exiting the capillary becomes divergent and eventually is not present within the central region where the collecting optical fibre is located. However the value of 10 degrees divergent incident beam is possible theoretically it is unlikely to occur in the real experimental setup. 


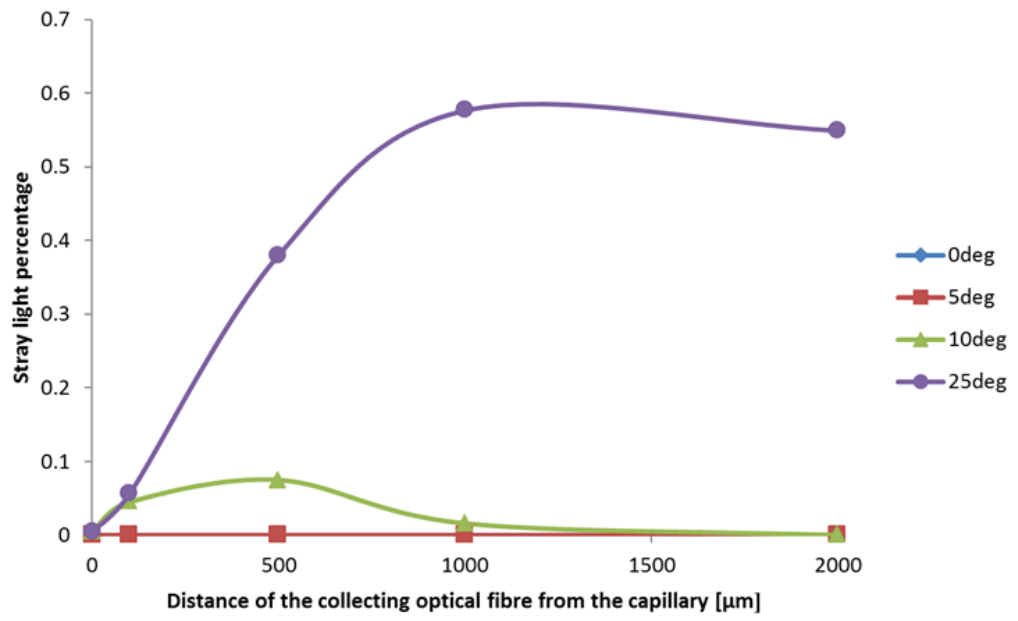

Figure 9. Percentage of stray light collected by a $100 \mu \mathrm{m}$ optical fibre related to its distance from capillary and for four different values of the incident beam divergence angle: 0, 5, 10 and 25 degrees.

The divergence value of 25 degrees as the divergence of the light exiting fused silica optical fibre was selected for other comparison - what diameter of the illuminating and collecting optical fibre should be used to maximise the amount of light interacting with the capillary content while minimising the amount of stray light. The values were modelled for $100 \mu \mathrm{m}$ internal diameter capillary. Two different approaches were tested:

- wide-area illumination and small area collection, attempting to collect only the light passing through the capillary bore.

- narrow-area illumination attempting only to illuminate the capillary bore and collecting as much light as possible

The results are summarised in Figure 10 and Figure 11. Figure 10 presents the collected light intensity that passed through the capillary bore and could interact with its content depending on the distance of the collecting fibre from the capillary.

Figure 11 presents percentage amount of stray light associated with the respective setting. The first number represents the illuminating optical fibre diameter in micrometres, and the second represents the collecting fibre diameter in micrometres as well.

The highest overall light intensity of the light able to interact with the capillary content was achieved with $50 \mu \mathrm{m}$ illuminating and $200 \mu \mathrm{m}$ collecting fibres. However at the position when maximum light intensity is collected the amount of stray light was unsatisfactory (close to 20\%). The large diameter of the collecting fibre, twice of the internal capillary diameter, was large enough for the light to propagate around the capillary bore and still enter the fibre under a sufficient angle to stay within the optical fibre. 


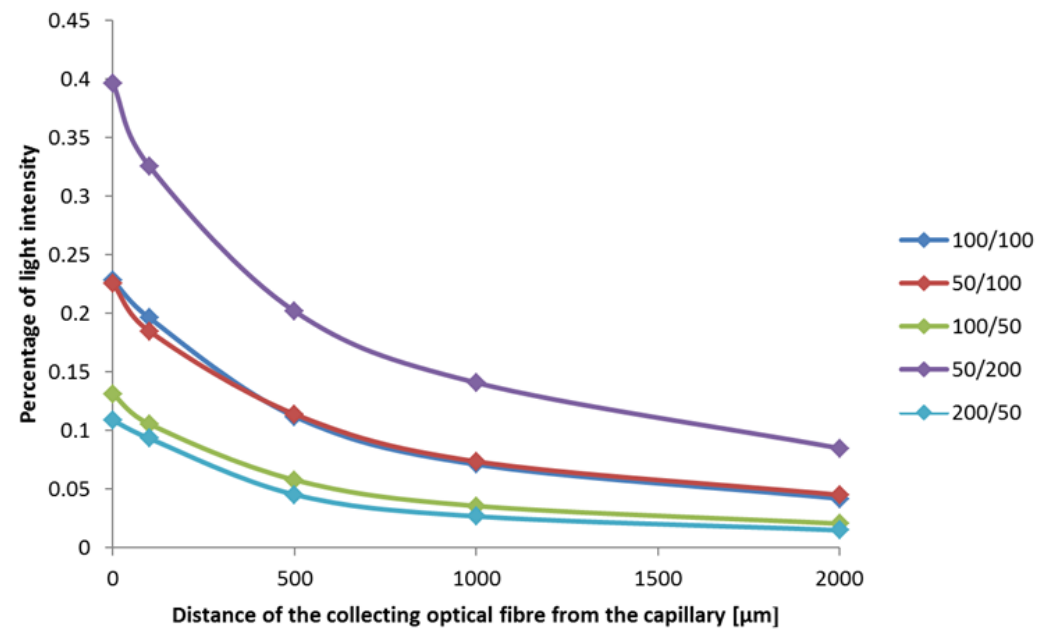

Figure 10. The amount of the initial light intensity that propagates through the capillary bore and subsequently is collected compared for different combinations of diamteres of the illuminating fibre (the first number) and the collecting fibre (the second number)

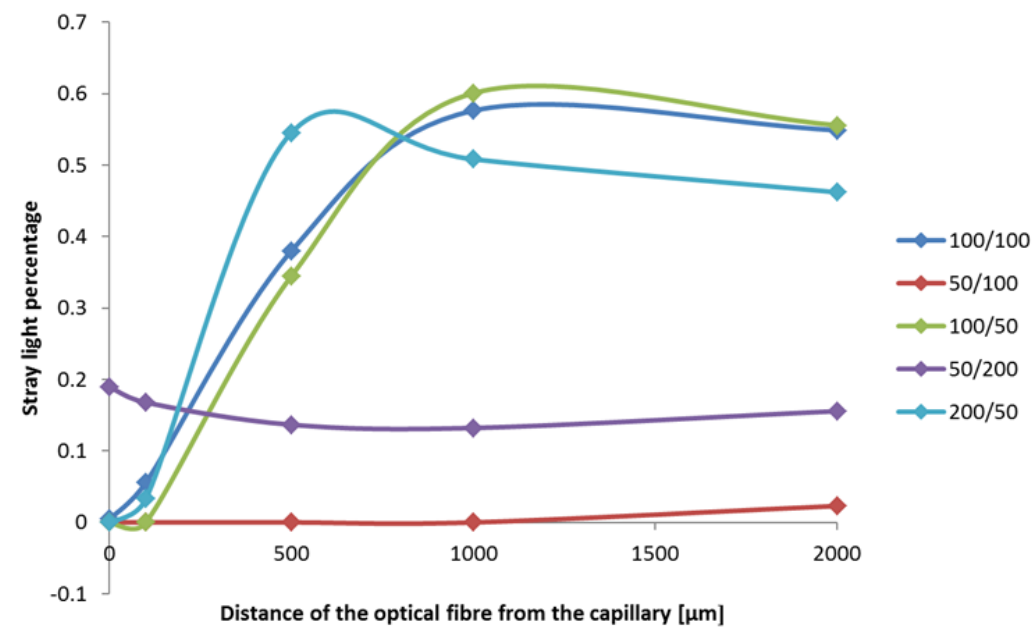

Figure 11. The amount of the stray light collected, compared for different combinations of diamteres of the illuminating fibre (the first number) and the collecting fibre (the second number)

Reducing the diameter of the collecting fibre to $100 \mu \mathrm{m}$ helped avoid stray light even when the distance between the collecting fibre and the capillary was around $1 \mathrm{~mm}$. This result can be very helpful as it allows for the high efficiency of the light collection combined with the minimisation of the stray light due to imprecise assembly of the detection system.

Combination of $100 \mu \mathrm{m}$ illuminating and $100 \mu \mathrm{m}$ collecting fibres gave good results in terms of the available light intensity, but even a slightest misalignment of the collecting optical 
fibre $(0.2 \mathrm{~mm}$ distance between can result in large amount of stray light delivered to the detector. Other combinations $(100 \mu \mathrm{m}$ and $200 \mu \mathrm{m}$ illuminating fibres and $50 \mu \mathrm{m}$ collecting fibre) produced significantly lower available light intensity combined with high vulnerability to stray light introduction due to non-perfect assembly of the optical system.

In order to design a capillary based photo detection system the following occurrences should be considered: effect of stray light, amount of light intensity within the capillary bore, location of illuminating and collecting optical fibres and diameters thereof, as well as their angle of incidence. When considering all of these factors in conjunction, a complex hyperplane of possible solutions presents itself which cannot be simply understood or calculated. It is therefore valuable to have a numerical model which can simultaneously take into account these factors. Such a model can also be used for other applications. An example of this is presented in the following sections for the application of photopolymerisation.

\section{Optimisation of the photochemical reactions in capillaries}

The main aim of this part is to present qualitative and semi-quantitative information about light behaviour in photopolymerisation setups. The capillary geometry and its multi-layered construction resulting in unusual and unexpected optical properties should be better understood as it allows to improve the work efficiency with capillaries. As explained in later part of this article many of described problems occur only in polytetrafluoroethylene (PTFE) coated capillaries.

For photoinitiated polymerisation in UV-range polyimide coated capillaries cannot be used because of polyimide high absorptivity below $550 \mathrm{~nm}$. PTFE-coated capillaries were selected as PTFE is transparent in UV region. During several experiments it was noted that position of LED versus capillary play significant role in shape and quality of formed polymer. When LED was placed too close to the capillary monolith was growing distance of several millimetres under photomask. It was also noted that edges of formed polymer were uneven and blurry. If LED tilted there was noticeable difference in growth of one end of monolith. Observed problems in polymerisation setup are presented schematically on Figure 12. A series of experiments was conducted to repeat mentioned observation and approach them qualitatively and semi-quantitatively. The experimental conditions for photopolymerisation reactions are presented elsewhere [14].

A series of experiments were conducted in order to evaluate the degree of monolith growth under the masked region of a capillary where direct light should not enter. Observed results in experiments with photopolymerisation using same mixture and light sources showed that monolith can grow few millimetres under the mask. Such growth is considered undesired. Table 1 and Figure 14 shows average of three results of photoinitiated polymerisation of monolith with measured distance of the part formed under the photomask - in a region where direct light should not reach and therefore polymerisation would not be anticipated or well controlled. There was a clear trend of increased length of monolith grown under the mask as the LED was placed closer to the capillary. Also when the LED was tilted by an angle of $45^{\circ}$ growth was significantly larger that side compared to when the LED was held 
perpendicularly. The light source was located directly over the edge of the photomask as shown in Figure 13.

(a)
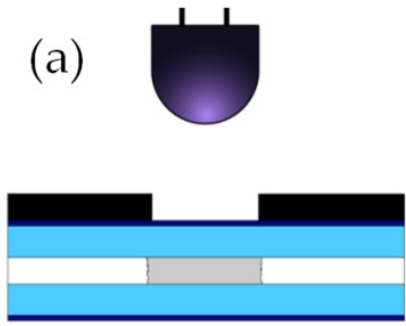

(b)
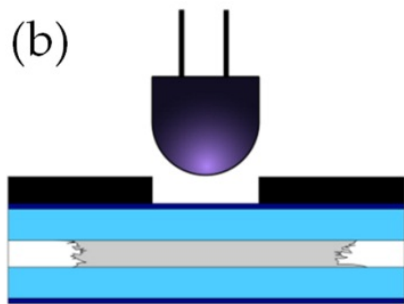

(c)
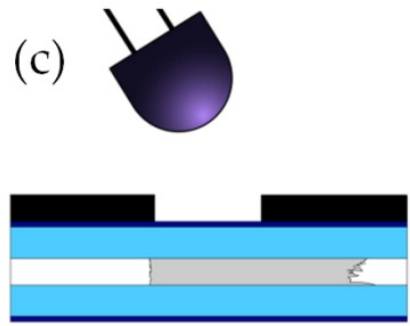

Figure 12. Schematic illustration of observed problems with monoliths obtained by photoinitiated polymerisation: (a) ideal monolith, (b) monolith growing up to several milimeters under the photomask in both direction when LED was too close to the capillary, (c) monolith growing on one end when the LED was tilted.
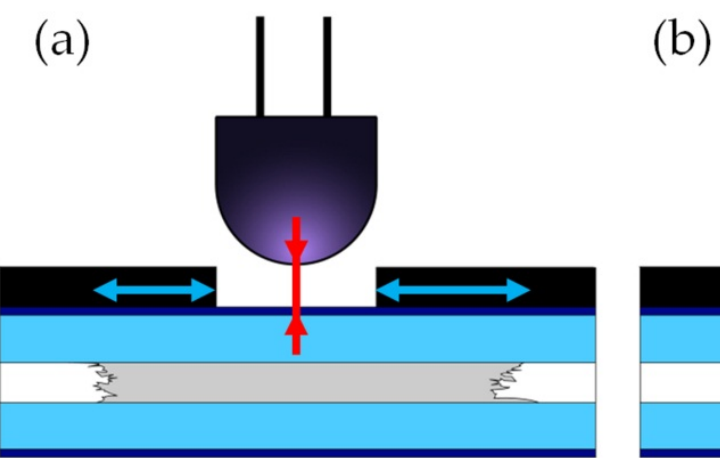

Figure 13. (a) schematic of the LED placement versus the capillary for perpendicular illumination and (b) for illumination under $45^{\circ}$ angle. Red arrow marks distance from capillary to the LED, blue arrows marks distance of the monolith growth.

\begin{tabular}{|c|c|c|c|}
\hline \multicolumn{2}{|c|}{ LED perpendicular } & \multicolumn{2}{c|}{ LED tilted by $45^{\circ}$} \\
\hline $\begin{array}{c}\text { LED distance from } \\
\text { capillary }\end{array}$ & Growth length & $\begin{array}{c}\text { LED distance from } \\
\text { capillary }\end{array}$ & Growth length \\
\hline $0 \mathrm{~cm}$ & $1.1 \mathrm{~mm}$ & $0 \mathrm{~cm}$ & $2.3 \mathrm{~mm}$ \\
$1 \mathrm{~cm}$ & $0.7 \mathrm{~mm}$ & $1 \mathrm{~cm}$ & $1.9 \mathrm{~mm}$ \\
$2.5 \mathrm{~cm}$ & $0.5 \mathrm{~mm}$ & $2.5 \mathrm{~cm}$ & $1.7 \mathrm{~mm}$ \\
$4 \mathrm{~cm}$ & $0 \mathrm{~mm}$ & $4 \mathrm{~cm}$ & $1.4 \mathrm{~mm}$ \\
\hline
\end{tabular}

Table 1. Monolith growth depending on light source position. 


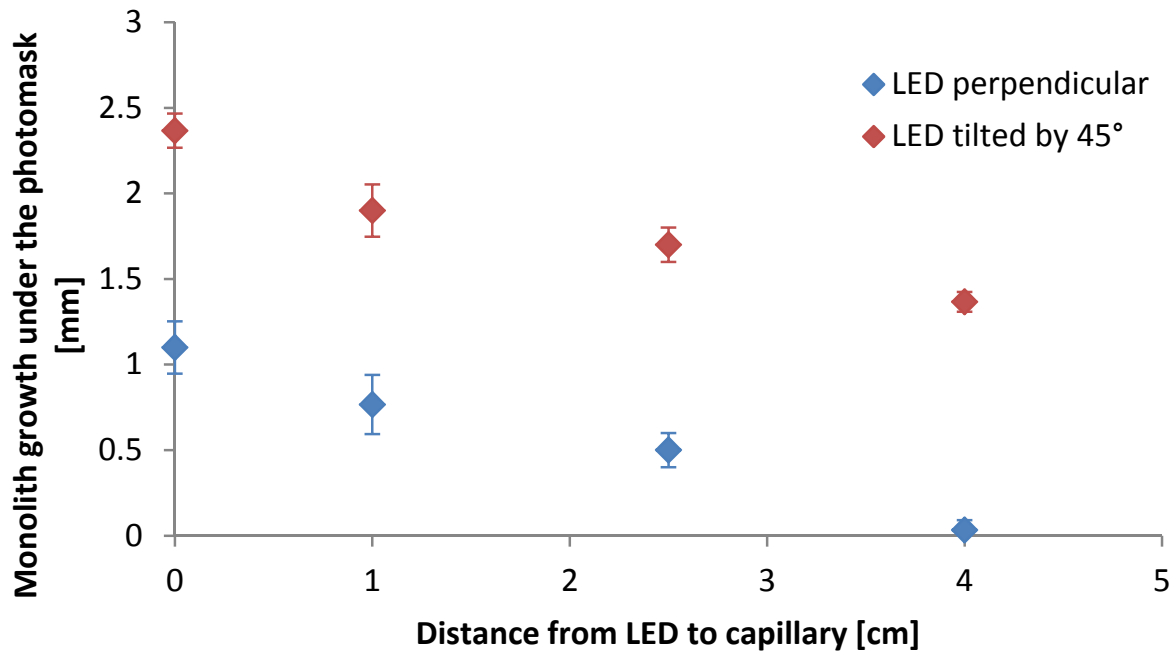

Figure 14. Relation between distance from the light source and monolith growth distance beyond the photomask covering for the case of the LED shining perpendicularly to the capillary and at an angle of $45^{\circ}$ to the capillary.

Previously described theoretical model for light propagation was used in order to understand what is happening during photopolymerisation with light, solution and monolith. Tilting of the LED and thus delivering significantly more light to one side of the monolith suggest that capillary geometry plays significant role.

When the light is incident on a boundary of two dielectrics with different dielectric constant (e.g. PTFE/fused silica, fused silica / polymerization mixture) a portion of it undergoes reflection reducing the intensity of transmitted light. The intensity of the reflected and transferred light is given by Fresnel's equations (Eq. 8) separately for each polarisation of light:

$$
R_{s}=\left[\frac{\sin \left(\theta_{i}-\theta_{t}\right)}{\sin \left(\theta_{i}+\theta_{t}\right)}\right]^{2} R_{p}=\left[\frac{\tan \left(\theta_{i}-\theta_{t}\right)}{\tan \left(\theta_{i}+\theta_{t}\right)}\right]^{2}
$$

The uncollimated light is mixture of all possible polarisations, that is combination of linear polarisations and can be separated for $p$ polarisation (vector of the electric field is parallel to plane of incidence) and s polarisation (vector of the electric field is perpendicular to plane of incidence). Graph on Figure 15 shows percentage of light being reflected on boundary PTFE/fused silica (light coming from medium with lower refractive index to medium with higher one) versus angle of incidence for both polarisations calculated from Eq. 8.

The light that reflects multiple times on dielectric boundary quickly loses intensity. The developed numerical model showed that incidence angles that are present in a capillary at the fused silica/PTFE boundary illuminated from outside do not provide significant 
reflectance and light is quickly transmitted outside of the capillary. Figure 16 provide information with upper limits of the light intensity that can be reflected by the capillary assuming total lack of absorption at this stage.

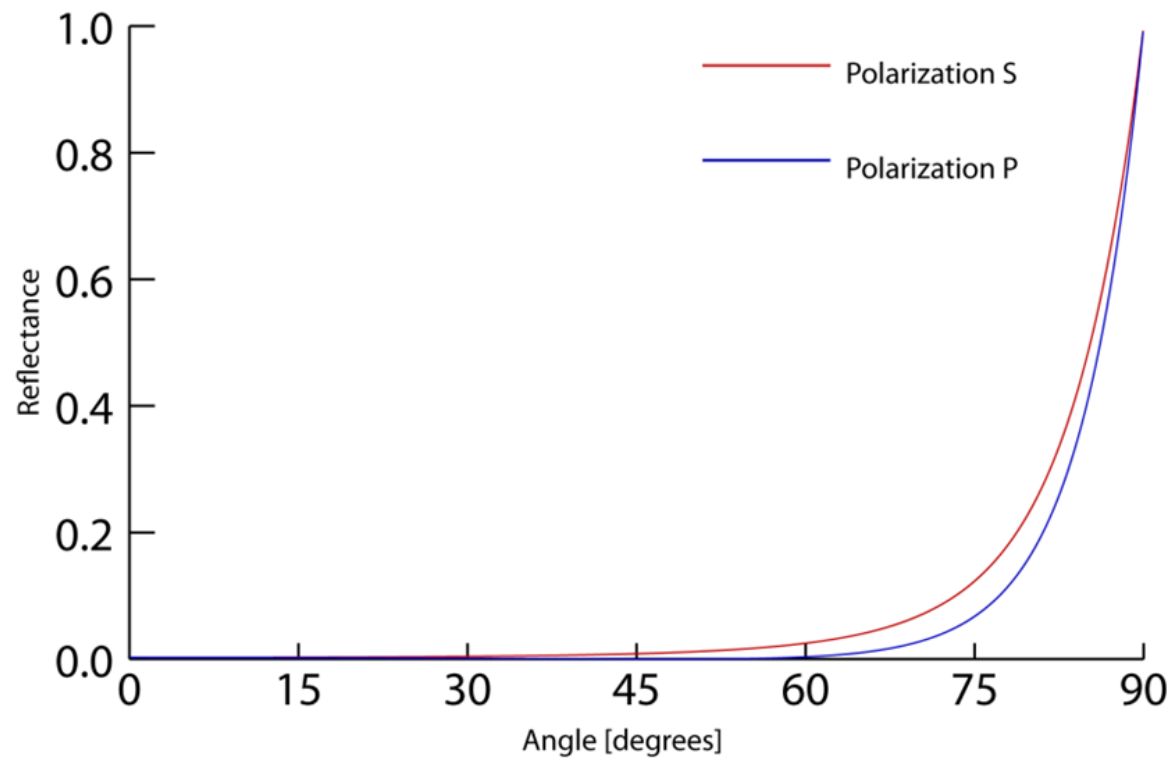

Figure 15. Theoretical reflectance of $s$ and $p$ polarised light incident on boundary PTFE/fused silica versus the angle of incidence; calculated from Eq. 8.

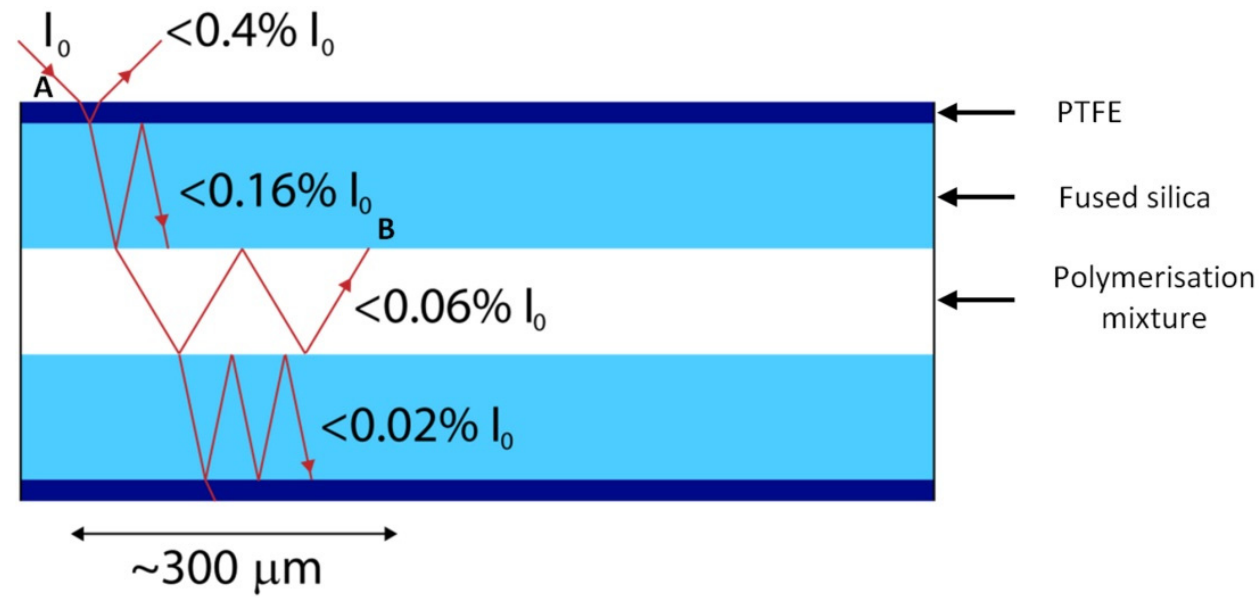

Figure 16. Schematic of the light propagation by multiple reflections inside PTFE-coated fused silica capillary with upper limit of the initial intensity Io (no more than). Dark blue - PTFE, light blue - fused silica, white - polymerisation mixture, red - light path 
Figure 16 shows schematic cross-section of capillary with sample light ray. After two reflections (first on boundary fused silica/polymerisation mixture, second on boundary fused silica/PTFE) not more than $0.16 \%$ of intensity from point $\mathrm{A}$ is delivered to point $\mathrm{B}$. The real value of the light intensity in point $\mathrm{A}$ is already lower than $\mathrm{I}$ due to reflection on boundary air/PTFE and boundary PTFE/fused silica, but these effects are neglected and rounded to Io is this discussion. After three reflections intensity drops to not more than $0.06 \%$ of initial, and after four to not more than $0.02 \%$. Dimensions of the capillary and refractive indices of PTFE, fused silica and polymerisation mixture are enforcing angles in further reflections and transmittances - after around $300 \mu \mathrm{m}$ from initial point of illumination total delivered light intensity is below $0.2 \%$ of the initial light intensity Io delivered to the capillary is available. This calculations are based on assumption that all materials (air, PTFE, fused silica and polymerisation mixture) are completely transparent and do not absorb any light. Because their transmission coefficients are below 1 total amount of light available will be significantly lower, making impossible to penetrate distances observed in the experiments.

The size of a standard capillary is comparable with size of multimode optical fibres, where geometrical optics is sufficient to explain observations and calculate results. Capillaries and coating have form of coaxial cylinders. The basic principle of optical (multimode) waveguide is total internal reflection (TIR). This phenomenon is occurring when dielectric with refractive index $\mathrm{n}_{1}$ (e.g. fused silica (FS)) is covered with dielectric with refractive index $\mathrm{n}_{2}<\mathrm{n}_{1}$ (e.g. PTFE, $\mathrm{nPTFE}_{\mathrm{n}}<\mathrm{nFs}$ ). The light wave incoming from medium with higher refractive index to boundary can undergo TIR provided angle of incidence is high enough. The graph on Figure 17 shows dependence of intensity of reflected light versus angle of incidence (for fused silica/PTFE boundary).

In order to observe total internal reflection for light incident on fused silica/PTFE boundary, angle of incidence must be higher than $64.8^{\circ}$, otherwise some light will be transmitted through the boundary resulting with loss of the light intensity. The developed numerical model showed that no angle higher than $44.7^{\circ}$ is available in any part of the capillary meaning that no light introduced to capillary from source placed above the capillary can achieve angle sufficient to reflect totally within the fused silica.

Capillaries are made of fused silica and have very similar diameters to optical fibres. PTFE has lower refractive index than fused silica. Initially a hypothesis about the capillary acting as an optical waveguide was discussed to explain occurrences of monolith growth under the photomask. There were two major contradictions: light coming from outside of cone of acceptance (i.e. from source located above the capillary) for optical fibre it cannot be transmitted over longer distance. The value of the highest possible angle of incidence on boundary fused silica/PTFE for light interacting with capillary content obtained from the developed model is significantly lower than required for the TIR. Graph on Figure 17 shows that for angles of incidence below $45^{\circ}$, reflectance is very low, and light is mostly transmitted through the boundary.

The last possibility was reflection on boundary PTFE/air - the highest possible ratio of refractive indices. In order to observe TIR on this boundary the incidence angle of light must 
not be lower than $49.25^{\circ}$. The highest incidence angle on fused silica/PTFE boundary calculated using light propagation model gives angle of $44.57^{\circ}$. Although amount of light reflected on that boundary is around $10 \%$ it does not satisfy the condition to observe total internal reflection and could not explain growth of few millimetres.

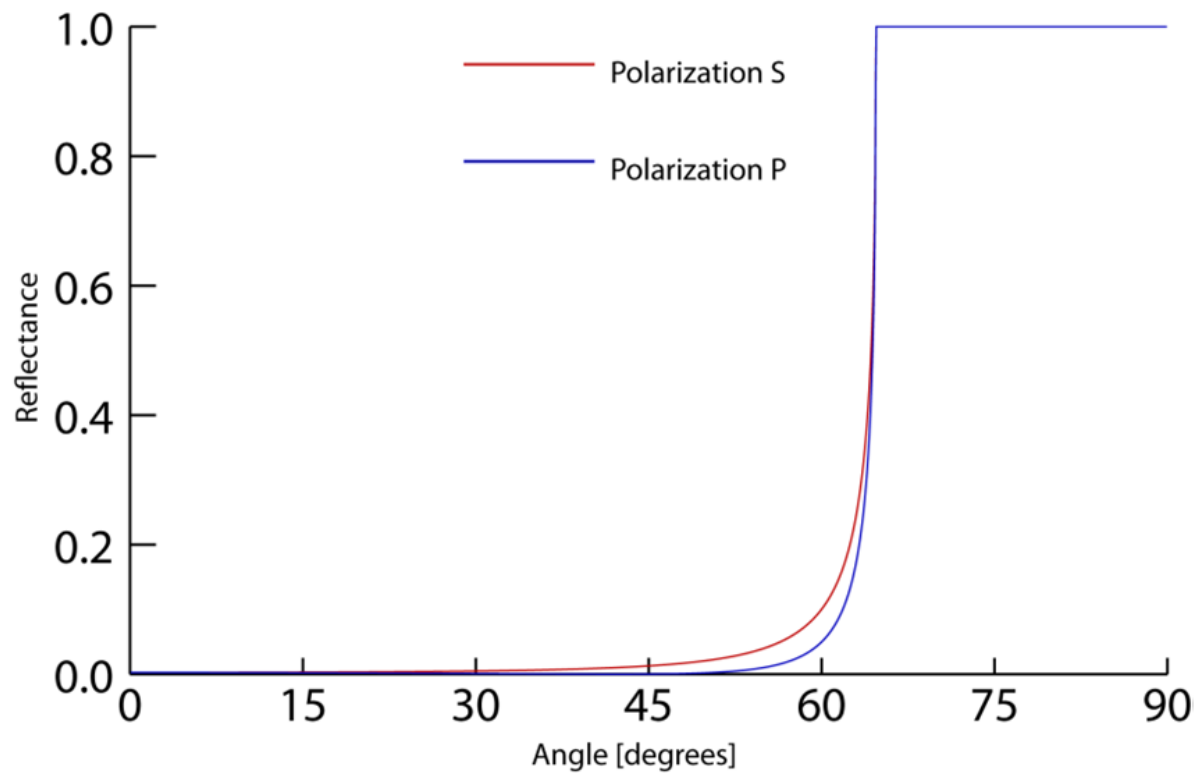

Figure 17. Theoretical reflectance on boundary fused silica/PTFE versus the angle of incidence.A reflectance value of one (starting at $64.8^{\circ}$ ) shows where total internal reflection occurs and no light is transmitted through the boundary (from Eq. 8).

These attempts mentioned above to explain observed result were based on a static system with constant time-independent properties. A capillary with on-going polymerisation reaction is a dynamic system, which changes its physical and optical properties in time. A hypothesis that a monolith forming inside capillary is changing optical properties of the setup during the polymerisation was posed. To prove this hypothesis, new photographs of capillary filled with polymerisation mixture and monolith were taken to show the transmission of light when the LED was shining on the capillary. The capillary was installed vertically above the digital microscope. In order to prevent any undesired light, a black cardboard separated the microscope objective from the rest of the setup and photos were taken in total darkness. Any possible openings near capillary wall were covered with a sealant. Schematic of that setup is shown on Figure 18.

It can be clearly seen in the Figure 19a that light was transmitted through the fused silica. The distance from the light source to the microscope was $20 \mathrm{~mm}$ to prevent any other discussed method than waveguiding to propagate light toward the end of capillary. To confirm that the

${ }^{1}$ Calculated from Eq. 22, refractive index of PTFE nPTFE=1.32 and refractive index of air nair $=1$ 
observed effect has nothing to do with collimation of the light the experiment was repeated using $532 \mathrm{~nm}$ green laser as light source. Result is shown on Figure 20. Black spots are effect of destructive interference of laser beam with itself after multiple reflections inside fused silica. LED light is non-collimated thus the interference effect was not observed.

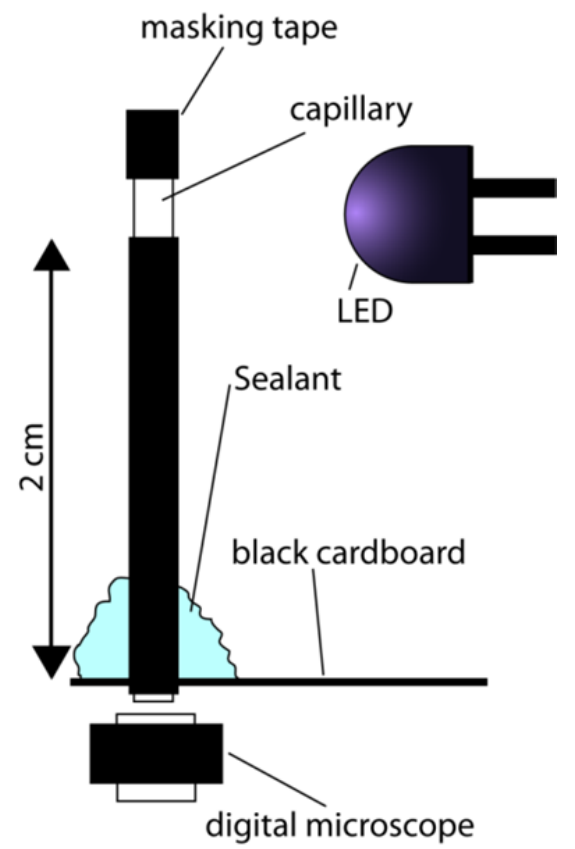

Figure 18. Schematic of experimental setup for observing light waveguiding inside the capillary with monolith.
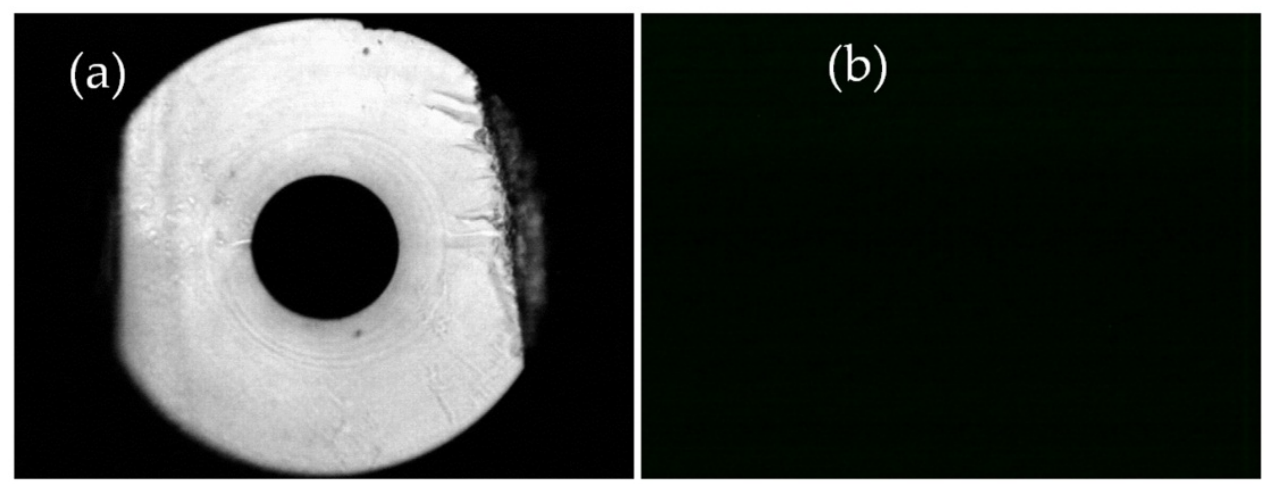

Figure 19. (a) Capillary with monolith and (b) empty capillary. Image collected by digital microscope using setup from Figure 18. 


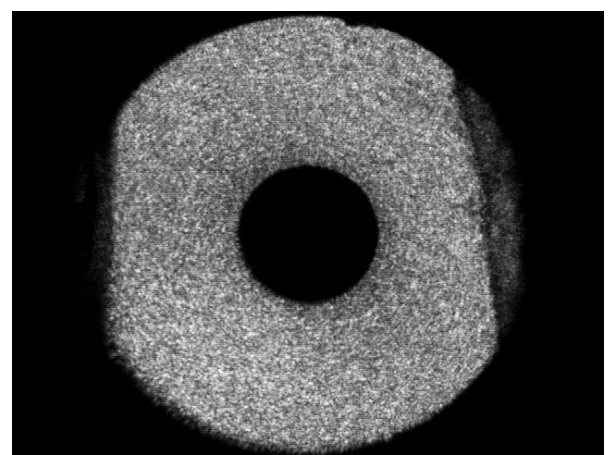

Figure 20. Image observed when $532 \mathrm{~nm}$ green laser is used as light source (setup same as in Figure 18).

The monolith inside the capillary has a very irregular surface. Moreover the refractive index of a polymer is higher than of the fused silica. The incident light was scattered on the monolith surface (polymerisation mixture/monolith boundary) and due to the morphology of the monolith surface it was scattered in all directions (Figure 21). This type of reflection is called diffuse reflection or diffuse scattering. In this situation light can be reflected under an angle sufficient to undergo total internal reflection on boundary fused silica/PTFE. These angles are not available for light that is not a subject to diffuse reflection. The refractive index of polymethacrylic polymer is higher than fused silica and ranges from 1.472 to 1.506 [15]. Light reflected diffusively in one part of monolith can propagate through the monolith, cross the boundary monolith/fused silica, and then remain in the TIR regime in regions where no monolith is present, effectively turning capillary into an optical waveguide.

Also diffuse scattering allows photons entering fused silica under angles higher than for those coming directly from light source. Wherever total internal reflection is occurring, on opposite side of boundary an evanescent field appears strong enough to initiate photopolymerisation. Figure 22 shows a schematic of that principle.

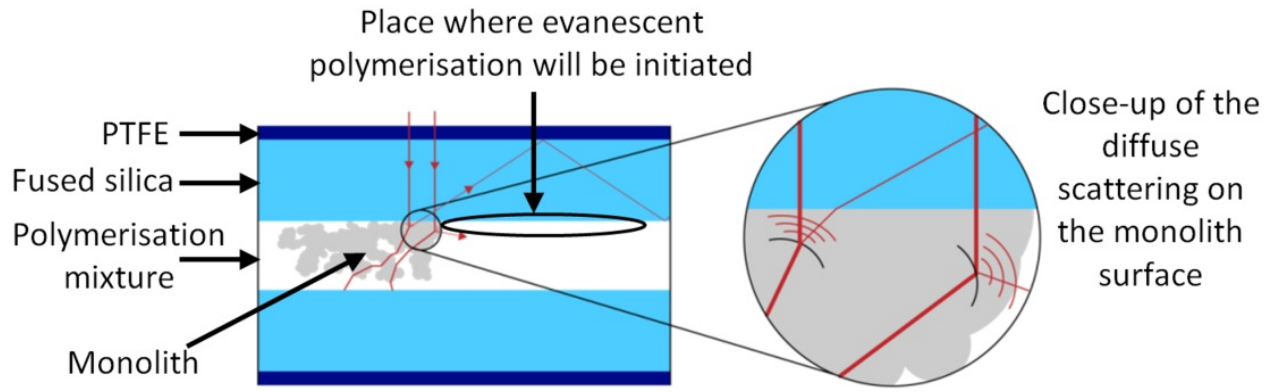

Figure 21. Schmatic of the diffuse scattering of incident light on the porous surface of the monolith that has formed inside the capillary. 


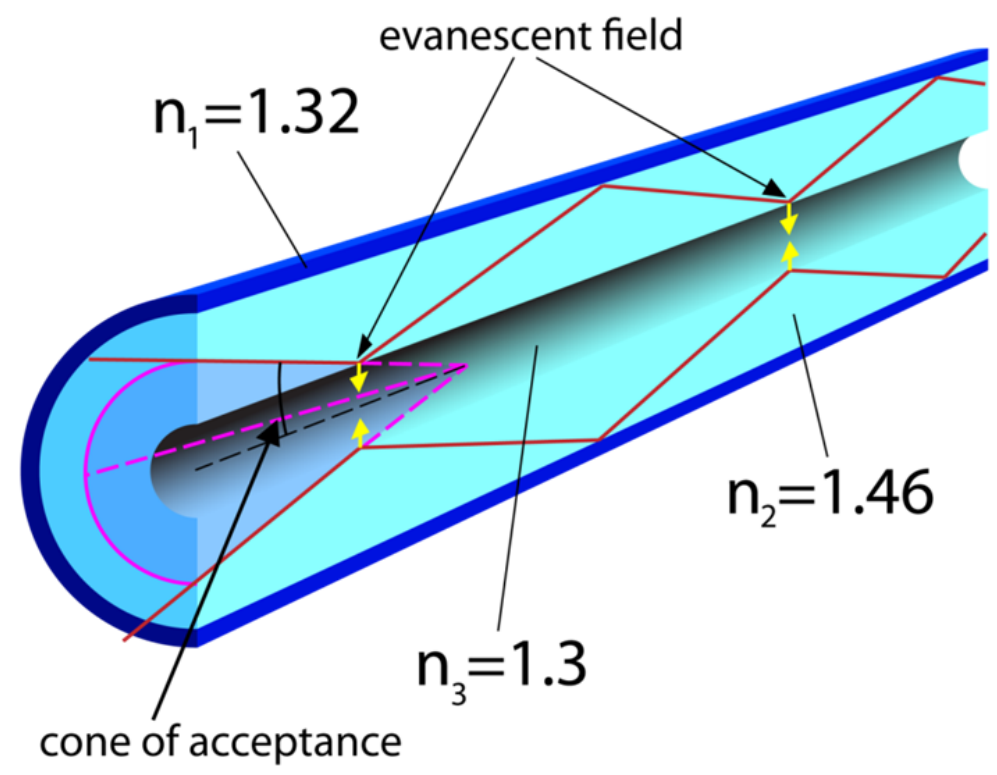

Figure 22. Schematic showing formation of the evanescent field (yellow arrow) outside dielectric inside which light undergoes total internal reflection. Dark blue - PTFE, light blue - fused silica, transparent polymerisation mixture.

\section{Conclusions}

The developed numerical model was used for investigation of the capillary optical properties during the photopolymerisation reaction. One of the important goals of this work was determining the relation between alignment of the light source and the shape and size of produced polymerised monoliths. Previous observations suggested that light transmission through the capillary fused silica body occurs in a similar manner as in an optical fibre. The numerical model was required to determine the conditions necessary for total internal reflection of the light used to initiate the polymerisation reaction, which in consequence would lead to waveguiding of the light through the capillary and enabled evanescent polymerisation in an obscured region under the photomask.

From the modelling and experimental results presented above, it can be seen that in cases where light is incident outside of the cone of acceptance, it cannot be confined by total internal reflection and thus does not propagate for significant distance. The presence of the photopolymerised monolith within the capillary, provides a surface for diffuse reflection, which in turn presents angles of internal reflection which would not otherwise be present. This enables the capillary to act as an optical waveguide.

The minimum required angle to observe total internal reflection without monolith was not present in the system. This result is concurrent with basic knowledge of the optical fibres: the light must be introduces into the waveguide under a sufficient angle to remain within 
total internal reflection regime. If the initial angle of incidence is higher than required the beam will eventually escape the waveguide. This hypothesis was tested using an empty capillary and a capillary filled with polymerisation mixture, which was illuminated perpendicularly. A photodetector was used to record the light emitted though the capillary cross-section. The results in both cases showed that there was no propagation of light along the capillary.

During this investigation the dynamics of the polymerisation process which are affected by the system optical properties was studied. The polymerisation process and the corresponding formation of a monolith inside the capillary would be expected to affect the optical properties of the whole system. An organic monolith is a highly porous, amorphic structure, that when illuminated reflects a significant amount of light. The presence of the monolith formed during the reaction changes the optical properties of the capillary that may no longer be regarded as a uniform cylindrical structure with constant refractive indices through the cross-section.

The monolith inside the capillary bore provided a surface for diffuse light reflection. The monolith's surface morphology allowed for presence of angles that are normally unavailable inside the capillary system, but with monolith waveguiding of the light becomes possible. The light can be transmitted along the capillary for significant distances (measured distance was $20 \mathrm{~mm}$ ) utilising the total internal reflection mechanism. When light is entering the capillary walls as in Figure 22 and undergoes total internal reflection within fused silica, an evanescent field will appear within the coating and the internal boundary of the capillary bore which is strong enough to initiate the reaction of photopolymerisation. Increased distance between the capillary and the light source significantly reduces the amount of light reflected from the monolith surface and thus the amount of light that can propagate through the capillary.

\section{Author details}

Tomasz Piasecki, Aymen Ben Azouz and Dermot Brabazon

School of Mechanical Engineering and Manufacturing, Dublin City University, Dublin, Ireland Irish Separation Science Cluster, Dublin, Ireland

Brett Paull and Mirek Macka

School of Chemistry, University of Tasmania, Hobart, Australia

\section{References}

[1] Capillary and microchip electrophoresis: Challenging the common conceptions. Breadmore, M. C. 2012, Journal of Chromatography A, Vol. 1221, pp. 42- 55.

[2] Rectangular Capillaries for Capillary Zone Electrophoresis. Tsuda, T., Sweedler, J. V., Zare, R. N. 1990, Analytical Chemistry, Vol. 62, pp. 2149-2152.

[3] Capillary Electrophoresis. Kuhr, W. G. 1990, Analytical Chemistry, Vol. 62, pp. 403R-414R. 
[4] Enhancement of detection sensitivity for indirect photometric detection of anions and cations in capillary electrophoresis. Johns, C., Macka, M., Haddad, P. R. 2003, Electrophoresis, Vol. 24, pp. 2150-2167.

[5] Skoog, D. A., Holler, F. J., Crouch, S. R. Principles of instrumental analysis. Belmont: Thomson Books/Cole, 2007. ISBN 13: 978-0-495-12570-9.

[6] Practical method for evaluation of linearity and effective pathlength of on-capillary photometric detectors in capillary electrophoresis. Johns, C., Macka, M., Haddad, P. R., King, M., Paull, B. 2001, Journal of Chromatography A, Vol. 927, pp. 237-241.

[7] Nanoliter-Scale Multireflection Cell for Absorption Detection in Capillary Electrophoresis. Wang T., Aiken, J. H., Huie, C. W., Hartwick, R. A. 1991, Analytical Chemistry, Vol. 63, pp. 1372-1376.

[8] Optical Improvements of a Z-Shaped Cell for High-Sensitivity UV Absorbance Detection in Capillary Electrophoresis. Moring, S., E., Reel, R. T. 1993, Analytical Chemistry, Vol. 65, pp. 3454-3459.

[9] On-column capillary flow cell utilizing optical wave-guide for chromatographic applications. Bruno, A. E., Gassmann, E., Pericles, N., Anton, K. 1989, Analytical Chemistry, Vol. 61, pp. 876-883.

[10] The pigtailing approach to optical detection in capillary electrophoresis. Bruno, A. E., Maystre, F., Krattiger, B., Nussbaum, P., Gassmann, E. 1994, Trends in Analytical Chemistry, Vol. 13, pp. 190-198.

[11] Numerical model for light propagation and light intensity distribution inside coated fused silica capillaries. Piasecki, T., Macka, M., Paull, B., Brabazon, D. 2011, Optics and Lasers in Enginnering, Vol. 49, pp. 924-931.

[12] Fiber-optic-based UV-visible absorbance detector for capillary electrophoresis, utilizing focusing optical elements. Lindberg, P., Hanning, A., Lindberg, T., Roeraade, J. 1998, Journal of Chromatography A, Vol. 809, pp. 181-189.

[13] Capillary Electrophoresis Detector Using a Light Emitting Diode and Optical Fibres. Butler, P. A. G., Mills, B., Hauser, P. 1997, Analyst, Vol. 122, pp. 949-953.

[14] UV-LED photopolymerised monoliths. Abele, S., Nie, F. Q., Foret, F., Paull, B., Macka, M. 2008, Analyst, Vol. 133, pp. 864-866.

[15] Brandrup, J., Immergut, E. H. Polymer Handbook. Ney York : John Wiley \& Sons, 1975. 